\title{
The space of maximal Fourier multipliers as a dual space
}

\author{
by \\ NaOhito Tomita (Osaka)
}

\begin{abstract}
Figà-Talamanca characterized the space of Fourier multipliers as the dual space of a certain Banach space. In this paper, we characterize the space of maximal Fourier multipliers as a dual space.
\end{abstract}

1. Introduction. Let $\mathcal{S}\left(\mathbb{R}^{n}\right)$ and $\mathcal{S}^{\prime}\left(\mathbb{R}^{n}\right)$ be the Schwartz spaces of all rapidly decreasing smooth functions and tempered distributions, respectively. The space $M_{p}\left(\mathbb{R}^{n}\right)$ of Fourier multipliers consists of all $m \in L^{\infty}\left(\mathbb{R}^{n}\right)$ such that $T_{m}$ is bounded on $L^{p}\left(\mathbb{R}^{n}\right)$, where $T_{m}$ is defined by $T_{m} f=\mathcal{F}^{-1}[m \widehat{f}]$ for $f \in \mathcal{S}\left(\mathbb{R}^{n}\right)$. We define the norm on $M_{p}\left(\mathbb{R}^{n}\right)$ by $\|m\|_{M_{p}}=\sup \left\|T_{m} f\right\|_{L^{p}}$, where the supremum is taken over all $f \in \mathcal{S}\left(\mathbb{R}^{n}\right)$ such that $\|f\|_{L^{p}}=1$. Let $C_{0}\left(\mathbb{R}^{n}\right)$ be the space of all continuous functions such that $\lim _{|x| \rightarrow \infty} f(x)=0$. For $1<p<\infty, p^{\prime}$ is the conjugate exponent of $p$ (that is, $1 / p+1 / p^{\prime}=1$ ). Let $\mathbb{Z}$ and $\mathbb{N}$ be the sets of all integers and positive integers, respectively. The space $A_{p}\left(\mathbb{R}^{n}\right)$ consists of all $f \in C_{0}\left(\mathbb{R}^{n}\right)$ which can be written as $f=\sum_{i \in \mathbb{N}} f_{i} * g_{i}$ in $L^{\infty}\left(\mathbb{R}^{n}\right)$, where $\left\{f_{i}\right\}_{i \in \mathbb{N}},\left\{g_{i}\right\}_{i \in \mathbb{N}} \subset \mathcal{S}\left(\mathbb{R}^{n}\right)$ and $\sum_{i \in \mathbb{N}}\left\|f_{i}\right\|_{L^{p}}\left\|g_{i}\right\|_{L^{p^{\prime}}}<\infty$. Then the norm $\|f\|_{A_{p}}$ is the infimum of the last sums over all representations of $f$.

In [6], Figà-Talamanca proved that $M_{p}\left(\mathbb{R}^{n}\right)=A_{p}\left(\mathbb{R}^{n}\right)^{*}$, where $A_{p}\left(\mathbb{R}^{n}\right)^{*}$ is the dual space of $A_{p}\left(\mathbb{R}^{n}\right)$ (see also Larsen [10]). Berkson, Paluszyński and Weiss applied Figà-Talamanca's result to wavelet theory [2] (for other applications, see Asmar, Berkson and Gillespie [1] and Figà-Talamanca and Gaudry [7]).

Maximal functions generated by Fourier multipliers were studied by, for example, Christ, Grafakos, Honzík and Seeger [3], Dappa and Trebels [4] and Kenig and Tomas [9]. For $m \in L^{\infty}\left(\mathbb{R}^{n}\right)$, the dyadic maximal Fourier

2000 Mathematics Subject Classification: 42B15, 42B25.

Key words and phrases: Figà-Talamanca's theorem, Fourier multipliers, maximal functions, translation invariant operators. 
multiplier operator $M_{m}$ is defined by

$$
M_{m} f(x)=\sup _{j \in \mathbb{Z}}\left|T_{m\left(2^{j} \cdot\right)} f(x)\right|=\sup _{j \in \mathbb{Z}}\left|\mathcal{F}^{-1}\left[m\left(2^{j} \cdot\right) \widehat{f}\right](x)\right|
$$

for $f \in \mathcal{S}\left(\mathbb{R}^{n}\right)$ ([3], [4]). We denote by $\max M_{p}\left(\mathbb{R}^{n}\right)$ the space of all $m \in$ $L^{\infty}\left(\mathbb{R}^{n}\right)$ such that $M_{m}$ is bounded on $L^{p}\left(\mathbb{R}^{n}\right)$. We define the norm on $\max M_{p}\left(\mathbb{R}^{n}\right)$ by

$$
\|m\|_{\max M_{p}}=\sup \left\{\left\|M_{m} f\right\|_{L^{p}}: f \in \mathcal{S}\left(\mathbb{R}^{n}\right),\|f\|_{L^{p}}=1\right\} .
$$

Then $\max M_{p}\left(\mathbb{R}^{n}\right)$ is a Banach space (Proposition 3.1). The purpose of this paper is to characterize $\max M_{p}\left(\mathbb{R}^{n}\right)$ as the dual space of a certain normed space. The space $\widetilde{A}_{p}\left(\mathbb{R}^{n}\right)$ consists of all $f \in C_{0}\left(\mathbb{R}^{n}\right)$ which can be written as

$$
f=\sum_{i \in \mathbb{N}} \sum_{j \in \mathbb{Z}} f_{i} * g_{i, j}\left(2^{j} \cdot\right) \quad \text { in } L^{\infty}\left(\mathbb{R}^{n}\right),
$$

where $\left\{f_{i}\right\}_{i \in \mathbb{N}},\left\{g_{i, j}\right\}_{i \in \mathbb{N}, j \in \mathbb{Z}} \subset \mathcal{S}\left(\mathbb{R}^{n}\right)$ and $\sum_{i \in \mathbb{N}} \sum_{j \in \mathbb{Z}}\left\|f_{i}\right\|_{L^{p}}\left\|g_{i, j}\right\|_{L^{p^{\prime}}}<\infty$. Note that, if the last condition is satisfied, then $\sum_{i \in \mathbb{N}} \sum_{j \in \mathbb{Z}} f_{i} * g_{i, j}\left(2^{j}\right.$.) $\in C_{0}\left(\mathbb{R}^{n}\right)$ and $\sum_{i \in \mathbb{N}}\left\|f_{i}\right\|_{L^{p}}\left\|\left\{g_{i, j}\right\}_{j \in \mathbb{Z}}\right\|_{L^{p^{\prime}}\left(\mathbb{R}^{n}, \ell^{1}(\mathbb{Z})\right)}<\infty$, where the norm $\left\|\left\{g_{j}\right\}_{j \in \mathbb{Z}}\right\|_{L^{p^{\prime}}\left(\mathbb{R}^{n}, \ell^{1}(\mathbb{Z})\right)}$ is, by definition, $\left\{\int_{\mathbb{R}^{n}}\left(\sum_{j \in \mathbb{Z}}\left|g_{j}(x)\right|\right)^{p^{\prime}} d x\right\}^{1 / p^{\prime}}$. We define the norm on $\widetilde{A}_{p}\left(\mathbb{R}^{n}\right)$ by

$$
\|f\|_{\widetilde{A}_{p}}=\inf \left\{\sum_{i \in \mathbb{N}}\left\|f_{i}\right\|_{L^{p}}\left\|\left\{g_{i, j}\right\}_{j \in \mathbb{Z}}\right\|_{L^{p^{\prime}}\left(\mathbb{R}^{n}, \ell^{1}(\mathbb{Z})\right)}: f=\sum_{i \in \mathbb{N}} \sum_{j \in \mathbb{Z}} f_{i} * g_{i, j}\left(2^{j} \cdot\right)\right\} .
$$

Then $\widetilde{A}_{p}\left(\mathbb{R}^{n}\right)$ is a normed space (Proposition 3.2 ). Also, $A_{p}\left(\mathbb{R}^{n}\right)$ is continuously embedded in $\widetilde{A}_{p}\left(\mathbb{R}^{n}\right)$. For $m \in \max M_{p}\left(\mathbb{R}^{n}\right)$, we define a linear functional $\varphi_{m}$ on $\widetilde{A}_{p}\left(\mathbb{R}^{n}\right)$ by

$$
\varphi_{m}(f)=\sum_{i \in \mathbb{N}} \sum_{j \in \mathbb{Z}} T_{m\left(2^{j} \cdot\right)} f_{i} * g_{i, j}(0)
$$

for $f=\sum_{i \in \mathbb{N}} \sum_{j \in \mathbb{Z}} f_{i} * g_{i, j}\left(2^{j} \cdot\right) \in \widetilde{A}_{p}\left(\mathbb{R}^{n}\right)$. We note that the right hand side of (1) is independent of the representation of $f$ (Lemma 3.6). Our main result is the following.

Theorem 1. Let $1<p<\infty$. If $m \in \max M_{p}\left(\mathbb{R}^{n}\right)$, then $\varphi_{m} \in \widetilde{A}_{p}\left(\mathbb{R}^{n}\right)^{*}$ and $\left\|\varphi_{m}\right\|_{\left(\widetilde{A}_{p}\right)^{*}}=\|m\|_{\max M_{p}}$. Conversely, if $\varphi \in \widetilde{A}_{p}\left(\mathbb{R}^{n}\right)^{*}$, then there exists $m \in \max M_{p}\left(\mathbb{R}^{n}\right)$ such that $\varphi=\varphi_{m}$. In this sense, $\max M_{p}\left(\mathbb{R}^{n}\right)=\widetilde{A}_{p}\left(\mathbb{R}^{n}\right)^{*}$.

2. Preliminaries. We define the Fourier transform $\mathcal{F} f$ and the inverse Fourier transform $\mathcal{F}^{-1} f$ of $f \in \mathcal{S}\left(\mathbb{R}^{n}\right)$ by

$$
\mathcal{F} f(\xi)=\widehat{f}(\xi)=\int_{\mathbb{R}^{n}} e^{-i \xi \cdot x} f(x) d x, \quad \mathcal{F}^{-1} f(x)=\frac{1}{(2 \pi)^{n}} \int_{\mathbb{R}^{n}} e^{i x \cdot \xi} f(\xi) d \xi .
$$


We also define the Fourier transform $\mathcal{F} u$ and the inverse Fourier transform $\mathcal{F}^{-1} u$ of $u \in \mathcal{S}^{\prime}\left(\mathbb{R}^{n}\right)$ by

$$
\langle\mathcal{F} u, \psi\rangle=\langle u, \mathcal{F} \psi\rangle, \quad\left\langle\mathcal{F}^{-1} u, \psi\right\rangle=\left\langle u, \mathcal{F}^{-1} \psi\right\rangle \quad \text { for all } \psi \in \mathcal{S}\left(\mathbb{R}^{n}\right) .
$$

Note that, if $u$ is an appropriate function, then $\langle u, \psi\rangle=\int_{\mathbb{R}^{n}} u(x) \psi(x) d x$. For $u \in \mathcal{S}^{\prime}\left(\mathbb{R}^{n}\right)$ and $\psi \in \mathcal{S}\left(\mathbb{R}^{n}\right)$, the convolution $u * \psi$ is defined by $u * \psi(x)=$ $\left\langle u, \tau_{x} \check{\psi}\right\rangle$, where $\tau_{x} \check{\psi}(y)=\check{\psi}(y-x)$ and $\check{\psi}(y)=\psi(-y)$. As usual, for a function $\psi$ on $\mathbb{R}^{n}$ and $t>0$, we write $\psi_{t}(x)=t^{-n} \psi(x / t)$.

The Hardy-Littlewood maximal operator $M$ is defined by

$$
M f(x)=\sup _{r>0} \frac{1}{|B(0, r)|} \int_{B(0, r)}|f(x-y)| d y
$$

for all locally integrable functions $f$ on $\mathbb{R}^{n}$, where $B(0, r)$ is the ball of radius $r$ centered at the origin and $|B(0, r)|$ denotes the Lebesgue measure of $B(0, r)$. The following lemma appears as [5, Proposition 2.7].

Lemma 2.1. Let $\psi$ be a function on $\mathbb{R}^{n}$ which is dominated by a nonnegative, radial, decreasing (as a function on $(0, \infty)$ ) and integrable function. Then there exists a constant $C>0$ such that

$$
\sup _{t>0}\left|\left(\psi_{t} * f\right)(x)\right| \leq C M f(x)
$$

for all locally integrable functions $f$.

3. Proofs. Throughout the rest of the paper, we always assume $1<$ $p<\infty$.

Proposition 3.1. $\max M_{p}\left(\mathbb{R}^{n}\right)$ is a Banach space.

Proof. We first check that $\|\cdot\|_{\max M_{p}}$ is a norm. Since $\|\cdot\|_{M_{p}} \leq\|\cdot\|_{\max M_{p}}$ and $\|\cdot\|_{L^{\infty}} \leq\|\cdot\|_{M_{p}}([8$, p. 217$])$, it follows that if $\|m\|_{\max M_{p}}=0$ then $m=0$. Let $m, m_{1}, m_{2} \in \max M_{p}\left(\mathbb{R}^{n}\right)$ and $\alpha \in \mathbb{C}$. Then $M_{\alpha m} f=|\alpha| M_{m} f$ and $M_{m_{1}+m_{2}} f \leq M_{m_{1}} f+M_{m_{2}} f$ give $\|\alpha m\|_{\max M_{p}}=|\alpha|\|m\|_{\max M_{p}}$ and $\left\|m_{1}+m_{2}\right\|_{\max M_{p}} \leq\left\|m_{1}\right\|_{\max M_{p}}+\left\|m_{2}\right\|_{\max M_{p}}$.

We next check that $\max M_{p}\left(\mathbb{R}^{n}\right)$ is complete. Let $\left\{m_{k}\right\} \subset \max M_{p}\left(\mathbb{R}^{n}\right)$ be a Cauchy sequence. Since $M_{p}\left(\mathbb{R}^{n}\right)$ is complete, and $\|\cdot\|_{M_{p}} \leq\|\cdot\|_{\max } M_{p}$, we see that there exists $m \in M_{p}\left(\mathbb{R}^{n}\right)$ such that $m_{k} \rightarrow m$ in $M_{p}\left(\mathbb{R}^{n}\right)$ as $k \rightarrow \infty$. From $\|\cdot\|_{L^{\infty}} \leq\|\cdot\|_{M_{p}}$ it follows that $m_{k} \rightarrow m$ in $L^{\infty}\left(\mathbb{R}^{n}\right)$ as $k \rightarrow \infty$. Hence, $m_{k} \rightarrow m$ in $\mathcal{S}^{\prime}\left(\mathbb{R}^{n}\right)$ as $k \rightarrow \infty$. Since $m_{k}\left(2^{j} \cdot\right) \rightarrow m\left(2^{j} \cdot\right)$ in $\mathcal{S}^{\prime}\left(\mathbb{R}^{n}\right)$ as $k \rightarrow \infty$ for all $j \in \mathbb{Z}$, we see that $T_{m_{k}\left(2^{j} \cdot\right)} f(x) \rightarrow T_{m\left(2^{j} \cdot\right)} f(x)$ as $k \rightarrow \infty$ for all $f \in \mathcal{S}\left(\mathbb{R}^{n}\right), x \in \mathbb{R}^{n}$ and $j \in \mathbb{Z}$. This gives

$$
\begin{aligned}
& \left|T_{m_{k}\left(2^{j} \cdot\right)} f(x)-T_{m\left(2^{j} \cdot\right)} f(x)\right|=\lim _{k^{\prime} \rightarrow \infty}\left|T_{m_{k}\left(2^{j} \cdot\right)} f(x)-T_{m_{k^{\prime}}\left(2^{j} \cdot\right)} f(x)\right| \\
& \quad=\liminf _{k^{\prime} \rightarrow \infty}\left|T_{m_{k}\left(2^{j \cdot}\right)} f(x)-T_{m_{k^{\prime}}\left(2^{j} \cdot\right)} f(x)\right| \leq \liminf _{k^{\prime} \rightarrow \infty} M_{m_{k}-m_{k^{\prime}}} f(x),
\end{aligned}
$$


so $M_{m_{k}-m} f \leq \liminf _{k^{\prime} \rightarrow \infty} M_{m_{k}-m_{k^{\prime}}} f$. On the other hand, since $\left\{m_{k}\right\}$ is a Cauchy sequence, for any $\varepsilon>0$ there exists $N \in \mathbb{N}$ such that

$$
\left\|m_{k}-m_{k^{\prime}}\right\|_{\max M_{p}}=\sup \left\|M_{m_{k}-m_{k^{\prime}}} f\right\|_{L^{p}}<\varepsilon
$$

for all $k, k^{\prime} \geq N$, where the supremum is taken over all $f \in \mathcal{S}\left(\mathbb{R}^{n}\right)$ such that $\|f\|_{L^{p}}=1$. Therefore, by Fatou's lemma, we get

$$
\left\|M_{m_{k}-m} f\right\|_{L^{p}} \leq\left\|\liminf _{k^{\prime} \rightarrow \infty} M_{m_{k}-m_{k^{\prime}}} f\right\|_{L^{p}} \leq \liminf _{k^{\prime} \rightarrow \infty}\left\|M_{m_{k}-m_{k^{\prime}}} f\right\|_{L^{p}} \leq \varepsilon
$$

for all $k \geq N$ and $f \in \mathcal{S}\left(\mathbb{R}^{n}\right)$ such that $\|f\|_{L^{p}}=1$. The proof is complete.

Proposition 3.2. $\widetilde{A}_{p}\left(\mathbb{R}^{n}\right)$ is a normed space.

Proof. We only prove that, if $f \in \widetilde{A}_{p}\left(\mathbb{R}^{n}\right)$ and $\|f\|_{\widetilde{A}_{p}}=0$, then $f=0$. We note that $\mathcal{S}\left(\mathbb{R}^{n}\right) \subset \max M_{p}\left(\mathbb{R}^{n}\right)$. Indeed, from Lemma 2.1 , for $\psi \in \mathcal{S}\left(\mathbb{R}^{n}\right)$ we have $M_{\psi} f(x) \leq C M f(x)$, where $M$ is the Hardy-Littlewood maximal operator (see Section 2). Since $M$ is bounded on $L^{p}\left(\mathbb{R}^{n}\right)([5$, Theorem 2.5]), we see that $M_{\psi}$ is bounded on $L^{p}\left(\mathbb{R}^{n}\right)$. Let $f \in \widetilde{A}_{p}\left(\mathbb{R}^{n}\right)$ and $\|f\|_{\widetilde{A}_{p}}=0$. For $\varepsilon>0$, we can find $\left\{f_{\varepsilon, i}\right\},\left\{g_{\varepsilon, i, j}\right\} \subset \mathcal{S}\left(\mathbb{R}^{n}\right)$ such that $f=$ $\sum_{i \in \mathbb{N}} \sum_{j \in \mathbb{Z}} f_{\varepsilon, i} * g_{\varepsilon, i, j}\left(2^{j} \cdot\right)$ in $L^{\infty}\left(\mathbb{R}^{n}\right), \quad \sum_{i \in \mathbb{N}}\left\|f_{\varepsilon, i}\right\|_{L^{p}}\left\|\left\{g_{\varepsilon, i, j}\right\}_{j}\right\|_{L^{p^{\prime}}\left(\mathbb{R}^{n}, \ell^{1}(\mathbb{Z})\right)}$ $<\varepsilon$ and $\sum_{i \in \mathbb{N}} \sum_{j \in \mathbb{Z}}\left\|f_{\varepsilon, i}\right\|_{L^{p}}\left\|g_{\varepsilon, i, j}\right\|_{L^{p^{\prime}}}<\infty$. Since $f \in C_{0}\left(\mathbb{R}^{n}\right)$, it is enough to prove that $\langle f, \psi\rangle=0$ for all $\psi \in \mathcal{S}\left(\mathbb{R}^{n}\right)$. Let $\psi \in \mathcal{S}\left(\mathbb{R}^{n}\right)$. Since

$$
\begin{aligned}
\langle f, \psi\rangle & =\sum_{i \in \mathbb{N}} \sum_{j \in \mathbb{Z}} \int_{\mathbb{R}^{n}} f_{\varepsilon, i} * g_{\varepsilon, i, j}\left(2^{j} x\right) \psi(x) d x \\
& =\sum_{i \in \mathbb{N}} \sum_{j \in \mathbb{Z}} \int_{\mathbb{R}^{n}}\left(\int_{\mathbb{R}^{n}} f_{\varepsilon, i}^{\vee}(y-x) g_{\varepsilon, i, j}(y) d y\right) \psi_{2^{j}}(x) d x \\
& =\sum_{i \in \mathbb{N}} \sum_{j \in \mathbb{Z}} \int_{\mathbb{R}^{n}} \psi_{2^{j}} * f_{\varepsilon, i}^{\vee}(y) g_{\varepsilon, i, j}(y) d y=\sum_{i \in \mathbb{N}} \sum_{j \in \mathbb{Z}} \int_{\mathbb{R}^{n}} T_{\widehat{\psi}\left(2^{j} \cdot\right)} f_{\varepsilon, i}^{\vee}(y) g_{\varepsilon, i, j}(y) d y
\end{aligned}
$$

we see that

$$
\begin{aligned}
|\langle f, \psi\rangle| & \leq \sum_{i \in \mathbb{N}} \int_{\mathbb{R}^{n}} M_{\widehat{\psi}} f_{\varepsilon, i}^{\vee}(y) \sum_{j \in \mathbb{Z}}\left|g_{\varepsilon, i, j}(y)\right| d y \\
& \leq \sum_{i \in \mathbb{N}}\left\|M_{\widehat{\psi}} f_{\varepsilon, i}^{\vee}\right\|_{L^{p}}\left\|\left\{g_{\varepsilon, i, j}\right\}_{j}\right\|_{L^{p^{\prime}}\left(\mathbb{R}^{n}, \ell^{1}(\mathbb{Z})\right)} \\
& \leq\|\widehat{\psi}\|_{\max } M_{p} \sum_{i \in \mathbb{N}}\left\|f_{\varepsilon, i}\right\|_{L^{p}}\left\|\left\{g_{\varepsilon, i, j}\right\}_{j}\right\|_{L^{p^{\prime}}\left(\mathbb{R}^{n}, \ell^{1}(\mathbb{Z})\right)}<\|\widehat{\psi}\|_{\max M_{p}} \varepsilon
\end{aligned}
$$

Hence, the arbitrariness of $\varepsilon$ gives $\langle f, \psi\rangle=0$. The proof is complete.

The following lemma appears as [8, (1.2)].

LEMMA 3.3. If $m \in M_{p}\left(\mathbb{R}^{n}\right)$, then $\|\psi * m\|_{M_{p}} \leq\|\psi\|_{L^{1}}\|m\|_{M_{p}}$ for all $\psi \in \mathcal{S}\left(\mathbb{R}^{n}\right)$. 
Lemma 3.4. If $m \in M_{p}\left(\mathbb{R}^{n}\right)$, then $\|\psi m\|_{M_{p}} \leq\left\|\mathcal{F}^{-1} \psi\right\|_{L^{1}}\|m\|_{M_{p}}$ for all $\psi \in \mathcal{S}\left(\mathbb{R}^{n}\right)$.

Proof. Use the fact that $T_{\psi m} f=\left[\mathcal{F}^{-1} \psi\right] * T_{m} f$.

Lemma 3.5. Let $m \in M_{p}\left(\mathbb{R}^{n}\right)$. If $\left\{f_{i}\right\}_{i \in \mathbb{N}},\left\{g_{i, j}\right\}_{i \in \mathbb{N}, j \in \mathbb{Z}} \subset \mathcal{S}\left(\mathbb{R}^{n}\right)$ satisfy $\sum_{i \in \mathbb{N}} \sum_{j \in \mathbb{Z}}\left\|f_{i}\right\|_{L^{p}}\left\|g_{i, j}\right\|_{L^{p^{\prime}}}<\infty$ and $\sum_{i \in \mathbb{N}} \sum_{j \in \mathbb{Z}} f_{i} * g_{i, j}\left(2^{j} \cdot\right)=0$ in $L^{\infty}\left(\mathbb{R}^{n}\right)$, then

$$
\sum_{i \in \mathbb{N}} \sum_{j \in \mathbb{Z}} T_{m\left(2^{j} \cdot\right)} f_{i} * g_{i, j}(0)=0 .
$$

Proof. Let $m \in M_{p}\left(\mathbb{R}^{n}\right)$ and $\psi$ be a $C^{\infty}\left(\mathbb{R}^{n}\right)$-function such that $\psi(\xi)=1$ if $|\xi| \leq 1, \psi(\xi)=0$ if $|\xi| \geq 2$. Also, let $\widetilde{\psi}$ be a radial $C^{\infty}\left(\mathbb{R}^{n}\right)$-function such that $\widetilde{\widetilde{\psi}}(\xi)=0$ if $|\xi| \geq 1$ and $\int_{\mathbb{R}^{n}} \widetilde{\psi}(\xi) d \xi=1$. Then we set $\varrho_{(\varepsilon)}=\psi(\varepsilon \cdot)\left[\widetilde{\psi}_{\varepsilon} * m\right]$ for $\varepsilon>0$, where $\widetilde{\psi}_{\varepsilon}=\varepsilon^{-n} \widetilde{\psi}(\cdot / \varepsilon)$. Since $\widetilde{\psi}_{\varepsilon} *[\psi(\varepsilon \cdot) f] \rightarrow f$ in $\mathcal{S}\left(\mathbb{R}^{n}\right)$ as $\varepsilon \rightarrow 0$ for all $f \in \mathcal{S}\left(\mathbb{R}^{n}\right)$, we see that

$$
\begin{aligned}
T_{\varrho_{(\varepsilon)}\left(2^{j} \cdot\right)} f * g(0) & =\left\langle\left[\mathcal{F}^{-1} \varrho_{(\varepsilon)}\right]_{2^{j}}, \check{f} * \check{g}\right\rangle=\left\langle\psi(\varepsilon \cdot)\left[\widetilde{\psi}_{\varepsilon} * m\right], \mathcal{F}^{-1}\left[\check{f} * \check{g}\left(2^{j} \cdot\right)\right]\right\rangle \\
& \rightarrow\left\langle m, \mathcal{F}^{-1}\left[\check{f} * \check{g}\left(2^{j} \cdot\right)\right]\right\rangle=T_{m\left(2^{j} \cdot\right)} f * g(0) \quad \text { as } \varepsilon \rightarrow 0
\end{aligned}
$$

for all $f, g \in \mathcal{S}\left(\mathbb{R}^{n}\right)$ and $j \in \mathbb{Z}$. Since $\|m(t \cdot)\|_{M_{p}}=\|m\|_{M_{p}}$ for all $t>0$, by Lemmas 3.3 and 3.4, we also have

$$
\begin{aligned}
\left\|\varrho_{(\varepsilon)}\left(2^{j} \cdot\right)\right\|_{M_{p}} & =\left\|\varrho_{(\varepsilon)}\right\|_{M_{p}} \leq\left\|\mathcal{F}^{-1}[\psi(\varepsilon \cdot)]\right\|_{L^{1}}\left\|\widetilde{\psi}_{\varepsilon} * m\right\|_{M_{p}} \\
& \leq\left\|\left[\mathcal{F}^{-1} \psi\right]_{\varepsilon}\right\|_{L^{1}}\left\|\widetilde{\psi}_{\varepsilon}\right\|_{L^{1}}\|m\|_{M_{p}}=\left\|\mathcal{F}^{-1} \psi\right\|_{L^{1}}\|\widetilde{\psi}\|_{L^{1}}\|m\|_{M_{p}} .
\end{aligned}
$$

This gives

$$
\left|T_{\varrho_{(\varepsilon)}\left(2^{j \cdot}\right)} f * g(0)\right| \leq\left\|\mathcal{F}^{-1} \psi\right\|_{L^{1}}\|\widetilde{\psi}\|_{L^{1}}\|m\|_{M_{p}}\|f\|_{L^{p}}\|g\|_{L^{p^{\prime}}}
$$

for all $f, g \in \mathcal{S}\left(\mathbb{R}^{n}\right)$ and $j \in \mathbb{Z}$. Let $\left\{f_{i}\right\}_{i \in \mathbb{N}},\left\{g_{i, j}\right\}_{i \in \mathbb{N}, j \in \mathbb{Z}} \subset \mathcal{S}\left(\mathbb{R}^{n}\right)$ satisfy $\sum_{i \in \mathbb{N}} \sum_{j \in \mathbb{Z}}\left\|f_{i}\right\|_{L^{p}}\left\|g_{i, j}\right\|_{L^{p^{\prime}}}<\infty$ and $\sum_{i \in \mathbb{N}} \sum_{j \in \mathbb{Z}} f_{i} * g_{i, j}\left(2^{j} \cdot\right)=0$ in $L^{\infty}\left(\mathbb{R}^{n}\right)$. By (2) and (3), we get

$$
T_{\varrho_{(\varepsilon)}\left(2^{j \cdot}\right)} f_{i} * g_{i, j}(0) \rightarrow T_{m\left(2^{j .}\right)} f_{i} * g_{i, j}(0) \quad \text { as } \varepsilon \rightarrow 0
$$

and

$$
\left|T_{\varrho_{(\varepsilon)}\left(2^{j} .\right)} f_{i} * g_{i, j}(0)\right| \leq\left\|\mathcal{F}^{-1} \psi\right\|_{L^{1}}\|\widetilde{\psi}\|_{L^{1}}\|m\|_{M_{p}}\left\|f_{i}\right\|_{L^{p}}\left\|g_{i, j}\right\|_{L^{p^{\prime}}}
$$

for each $i \in \mathbb{N}$ and $j \in \mathbb{Z}$. Hence, by the Lebesgue dominated convergence theorem, we get

$$
\lim _{\varepsilon \rightarrow 0} \sum_{i \in \mathbb{N}} \sum_{j \in \mathbb{Z}} T_{\varrho_{(\varepsilon)}\left(2^{j \cdot}\right)} f_{i} * g_{i, j}(0)=\sum_{i \in \mathbb{N}} \sum_{j \in \mathbb{Z}} T_{m\left(2^{j \cdot}\right)} f_{i} * g_{i, j}(0) .
$$

Since $\mathcal{F}^{-1} \varrho_{(\varepsilon)} \in L^{1}\left(\mathbb{R}^{n}\right)$ and $\sum_{i \leq N} \sum_{|j| \leq N} f_{i} * g_{i, j}\left(2^{j} \cdot\right) \rightarrow 0$ in $L^{\infty}\left(\mathbb{R}^{n}\right)$ as 
$N \rightarrow \infty$, we see that

$$
\begin{aligned}
\sum_{i \in \mathbb{N}} \sum_{j \in \mathbb{Z}} T_{\varrho_{(\varepsilon)}\left(2^{j} .\right)} f_{i} * & g_{i, j}(0)=\lim _{N \rightarrow \infty} \sum_{i \leq N} \sum_{|j| \leq N} \int_{\mathbb{R}^{n}}\left[\mathcal{F}^{-1} \varrho_{(\varepsilon)}\right](-x) f_{i} * g_{i, j}\left(2^{j} x\right) d x \\
& =\lim _{N \rightarrow \infty} \int_{\mathbb{R}^{n}}\left[\mathcal{F}^{-1} \varrho_{(\varepsilon)}\right](-x)\left(\sum_{i \leq N} \sum_{|j| \leq N} f_{i} * g_{i, j}\left(2^{j} x\right)\right) d x=0 .
\end{aligned}
$$

This completes the proof.

Lemma 3.6. Let $m \in M_{p}\left(\mathbb{R}^{n}\right)$. Then we can define a linear functional $\varphi_{m}$ on $\widetilde{A}_{p}\left(\mathbb{R}^{n}\right)$ by $(1)$.

Proof. To define $\varphi_{m}$, we need to show that, if $\left\{f_{i}^{(1)}\right\},\left\{f_{i}^{(2)}\right\},\left\{g_{i, j}^{(1)}\right\},\left\{g_{i, j}^{(2)}\right\}$ $\subset \mathcal{S}\left(\mathbb{R}^{n}\right)$ satisfy $\sum_{i \in \mathbb{N}} \sum_{j \in \mathbb{Z}}\left\|f_{i}^{(1)}\right\|_{L^{p}}\left\|g_{i, j}^{(1)}\right\|_{L^{p^{\prime}}}, \sum_{i \in \mathbb{N}} \sum_{j \in \mathbb{Z}}\left\|f_{i}^{(2)}\right\|_{L^{p}}\left\|g_{i, j}^{(2)}\right\|_{L^{p^{\prime}}}$ $<\infty$ and $\sum_{i \in \mathbb{N}} \sum_{j \in \mathbb{Z}} f_{i}^{(1)} * g_{i, j}^{(1)}\left(2^{j} \cdot\right)=\sum_{i \in \mathbb{N}} \sum_{j \in \mathbb{Z}} f_{i}^{(2)} * g_{i, j}^{(2)}\left(2^{j} \cdot\right)$ in $L^{\infty}\left(\mathbb{R}^{n}\right)$, then

$$
\sum_{i \in \mathbb{N}} \sum_{j \in \mathbb{Z}} T_{m\left(2^{j \cdot}\right)} f_{i}^{(1)} * g_{i, j}^{(1)}(0)=\sum_{i \in \mathbb{N}} \sum_{j \in \mathbb{Z}} T_{m\left(2^{j .}\right)} f_{i}^{(2)} * g_{i, j}^{(2)}(0) .
$$

To do this, we define $\left\{f_{i}^{(3)}\right\}_{i},\left\{\left\{g_{i, j}^{(3)}\right\}_{j}\right\}_{i} \subset \mathcal{S}\left(\mathbb{R}^{n}\right)$ by $\left\{f_{i}^{(3)}\right\}_{i}=\left\{f_{1}^{(1)}, f_{1}^{(2)}\right.$, $\left.f_{2}^{(1)}, f_{2}^{(2)}, \ldots\right\}$, and $\left\{\left\{g_{i, j}^{(3)}\right\}_{j}\right\}_{i}=\left\{\left\{g_{1, j}^{(1)}\right\}_{j},\left\{-g_{1, j}^{(2)}\right\}_{j},\left\{g_{2, j}^{(1)}\right\}_{j},\left\{-g_{2, j}^{(2)}\right\}_{j}, \ldots\right\}$. Then we have

$$
\begin{aligned}
& \sum_{i \in \mathbb{N}} \sum_{j \in \mathbb{Z}}\left\|f_{i}^{(3)}\right\|\left\|_{L^{p}}\right\| g_{i, j}^{(3)} \|_{L^{p^{\prime}}} \\
&=\sum_{i \in \mathbb{N}} \sum_{j \in \mathbb{Z}}\left\|f_{i}^{(1)}\right\|_{L^{p}}\left\|g_{i, j}^{(1)}\right\|_{L^{p^{\prime}}}+\sum_{i \in \mathbb{N}} \sum_{j \in \mathbb{Z}}\left\|f_{i}^{(2)}\right\|_{L^{p}}\left\|g_{i, j}^{(2)}\right\|_{L^{p^{\prime}}}<\infty
\end{aligned}
$$

and

$$
\sum_{i \in \mathbb{N}} \sum_{j \in \mathbb{Z}} f_{i}^{(3)} * g_{i, j}^{(3)}\left(2^{j} \cdot\right)=\sum_{i \in \mathbb{N}} \sum_{j \in \mathbb{Z}} f_{i}^{(1)} * g_{i, j}^{(1)}\left(2^{j} \cdot\right)-\sum_{i \in \mathbb{N}} \sum_{j \in \mathbb{Z}} f_{i}^{(2)} * g_{i, j}^{(2)}\left(2^{j} \cdot\right)=0 .
$$

Hence, by Lemma 3.5, we get

$$
\begin{aligned}
& \sum_{i \in \mathbb{N}} \sum_{j \in \mathbb{Z}} T_{m\left(2^{j .}\right)} f_{i}^{(1)} * g_{i, j}^{(1)}(0)-\sum_{i \in \mathbb{N}} \sum_{j \in \mathbb{Z}} T_{m\left(2^{j .}\right)} f_{i}^{(2)} * g_{i, j}^{(2)}(0) \\
&=\sum_{i \in \mathbb{N}} \sum_{j \in \mathbb{Z}} T_{m\left(2^{j} \cdot\right)} f_{i}^{(3)} * g_{i, j}^{(3)}(0)=0 .
\end{aligned}
$$

Thus, the values $\sum_{i \in \mathbb{N}} \sum_{j \in \mathbb{Z}} T_{m\left(2^{j} .\right)} f_{i} * g_{i, j}(0)$ are independent of the representations of $f$. In the same way, we can prove the linearity of $\varphi_{m}$.

We are now ready to prove Theorem 1 given in the introduction.

Proof of Theorem 1. We first prove that, if $m \in \max M_{p}\left(\mathbb{R}^{n}\right)$, then $\varphi_{m} \in \widetilde{A}_{p}\left(\mathbb{R}^{n}\right)^{*}$ and $\|m\|_{\max M_{p}}=\left\|\varphi_{m}\right\|_{\left(\widetilde{A}_{p}\right)^{*}}$. Let $m \in \max M_{p}\left(\mathbb{R}^{n}\right)$. By 
Lemma 3.6, we see that $\varphi_{m}$ is a linear functional on $\widetilde{A}_{p}\left(\mathbb{R}^{n}\right)$. Let $f=$ $\sum_{i \in \mathbb{N}} \sum_{j \in \mathbb{Z}} f_{i} * g_{i, j}\left(2^{j} \cdot\right) \in \widetilde{A}_{p}\left(\mathbb{R}^{n}\right)$. Since

$$
\begin{aligned}
\left|\varphi_{m}(f)\right| & \leq \sum_{i \in \mathbb{N}} \int_{\mathbb{R}^{n}} \sum_{j \in \mathbb{Z}}\left|T_{m\left(2^{j} \cdot\right)} f_{i}(x) g_{i, j}(-x)\right| d x \\
& \leq \sum_{i \in \mathbb{N}}\left\|M_{m} f_{i}\right\|_{L^{p}}\left\|\left\{g_{i, j}\right\}_{j}\right\|_{L^{p^{\prime}}\left(\mathbb{R}^{n}, \ell^{1}(\mathbb{Z})\right)} \\
& \leq\|m\|_{\max M_{p}} \sum_{i \in \mathbb{N}}\left\|f_{i}\right\|_{L^{p}}\left\|\left\{g_{i, j}\right\}_{j}\right\|_{L^{p^{\prime}}\left(\mathbb{R}^{n}, \ell^{1}(\mathbb{Z})\right)}
\end{aligned}
$$

taking the infimum over all the representations of $f$, we have $\left|\varphi_{m}(f)\right| \leq$ $\|m\|_{\max M_{p}}\|f\|_{\widetilde{A}_{p}}$, so $\varphi_{m} \in \widetilde{A}_{p}\left(\mathbb{R}^{n}\right)^{*}$ and $\left\|\varphi_{m}\right\|_{\left(\widetilde{A}_{p}\right)^{*}} \leq\|m\|_{\max M_{p}}$. To prove $\left\|\varphi_{m}\right\|_{\left(\widetilde{A}_{p}\right)^{*}} \geq\|m\|_{\max M_{p}}$, we use the duality $L^{p^{\prime}}\left(\mathbb{R}^{n}, \ell^{1}(\mathbb{Z})\right)^{*}=L^{p}\left(\mathbb{R}^{n}, \ell^{\infty}(\mathbb{Z})\right)$ ([12, Proposition, 2.11.1]), that is,

$$
\begin{aligned}
\|m\|_{\max M_{p}} & =\sup \left\|\left\{T_{m\left(2^{j} \cdot\right)} f\right\}_{j \in \mathbb{Z}}\right\|_{L^{p}\left(\mathbb{R}^{n}, \ell^{\infty}(\mathbb{Z})\right)} \\
& =\sup \left|\int_{\mathbb{R}^{n}} \sum_{j \in \mathbb{Z}} T_{m\left(2^{j} \cdot\right)} f(x) g_{j}(x) d x\right|,
\end{aligned}
$$

where the supremum is taken over all $f \in \mathcal{S}\left(\mathbb{R}^{n}\right)$ and finitely supported sequences $\left\{g_{j}\right\}_{j \in \mathbb{Z}} \subset \mathcal{S}\left(\mathbb{R}^{n}\right)$ such that $\|f\|_{L^{p}}=\left\|\left\{g_{j}\right\}_{j \in \mathbb{Z}}\right\|_{L^{p^{\prime}}\left(\mathbb{R}^{n}, \ell^{1}(\mathbb{Z})\right)}=1$. For $\varepsilon>0$, we can find $f_{\varepsilon} \in \mathcal{S}\left(\mathbb{R}^{n}\right)$ and a finitely supported sequence $\left\{g_{\varepsilon, j}\right\} \subset$

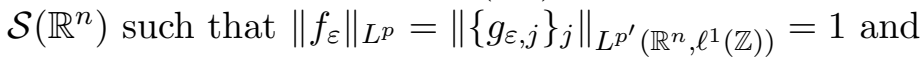

$$
\|m\|_{\max M_{p}}-\varepsilon<\left|\int_{\mathbb{R}^{n}} \sum_{j \in \mathbb{Z}} T_{m\left(2^{j} .\right)} f_{\varepsilon}(x) g_{\varepsilon, j}(x) d x\right| .
$$

Since $\left\{g_{\varepsilon, j}\right\} \subset \mathcal{S}\left(\mathbb{R}^{n}\right)$ is a finitely supported sequence, we have $\sum_{j \in \mathbb{Z}} f_{\varepsilon} *$ $g_{\varepsilon, j}^{\vee}\left(2^{j} \cdot\right) \in \widetilde{A}_{p}\left(\mathbb{R}^{n}\right)$ and $\left\|\sum_{j \in \mathbb{Z}} f_{\varepsilon} * g_{\varepsilon, j}^{\vee}\left(2^{j} \cdot\right)\right\|_{\widetilde{A}_{p}} \leq\left\|f_{\varepsilon}\right\|_{L^{p}}\left\|\left\{g_{\varepsilon, j}^{\vee}\right\}_{j}\right\|_{L^{p^{\prime}}\left(\mathbb{R}^{n}, \ell^{1}(\mathbb{Z})\right)}$. Hence, we get

$$
\begin{aligned}
\|m\|_{\max M_{p}} & <\left|\int_{\mathbb{R}^{n}} \sum_{j \in \mathbb{Z}} T_{m\left(2^{j} \cdot\right)} f_{\varepsilon}(x) g_{\varepsilon, j}(x) d x\right|+\varepsilon \\
& =\left|\sum_{j \in \mathbb{Z}} T_{m\left(2^{j} \cdot\right)} f_{\varepsilon} * g_{\varepsilon, j}^{\vee}(0)\right|=\left|\varphi_{m}\left(\sum_{j \in \mathbb{Z}} f_{\varepsilon} * g_{\varepsilon, j}^{\vee}\left(2^{j} \cdot\right)\right)\right|+\varepsilon \\
& \leq\left\|\varphi_{m}\right\|_{\left(\widetilde{A}_{p}\right)^{*}}\left\|\sum_{j \in \mathbb{Z}} f_{\varepsilon} * g_{\varepsilon, j}^{\vee}\left(2^{j} \cdot\right)\right\|_{\widetilde{A}_{p}}+\varepsilon \leq\left\|\varphi_{m}\right\|_{\left(\widetilde{A}_{p}\right)^{*}}+\varepsilon .
\end{aligned}
$$

Hence, the arbitrariness of $\varepsilon$ gives $\left\|\varphi_{m}\right\|_{\left(\widetilde{A}_{p}\right)^{*}} \geq\|m\|_{\max M_{p}}$.

We next prove that, if $\varphi \in \widetilde{A}_{p}\left(\mathbb{R}^{n}\right)^{*}$, then there exists $m \in \max M_{p}\left(\mathbb{R}^{n}\right)$ such that $\varphi=\varphi_{m}$. We note that, if $f, g \in \mathcal{S}\left(\mathbb{R}^{n}\right)$ and $j \in \mathbb{Z}$, then $f * g\left(2^{j}.\right) \in$ $\widetilde{A}_{p}\left(\mathbb{R}^{n}\right)$ and $\left\|f * g\left(2^{j} \cdot\right)\right\|_{\widetilde{A}_{p}} \leq\|f\|_{L^{p}}\|g\|_{L^{p^{\prime}}}$. For $f \in \mathcal{S}\left(\mathbb{R}^{n}\right)$ and $j \in \mathbb{Z}$, we can 
define a linear functional $L_{f}^{(j)}$ on the dense subspace $\mathcal{S}\left(\mathbb{R}^{n}\right)$ of $L^{p^{\prime}}\left(\mathbb{R}^{n}\right)$ by $L_{f}^{(j)}(g)=\varphi\left(f * g\left(2^{j} \cdot\right)\right)$ for $g \in \mathcal{S}\left(\mathbb{R}^{n}\right)$. Since

$$
\left|L_{f}^{(j)}(g)\right|=\left|\varphi\left(f * g\left(2^{j} \cdot\right)\right)\right| \leq\|\varphi\|_{\left(\widetilde{A}_{p}\right)^{*}}\left\|f * g\left(2^{j} \cdot\right)\right\|_{\widetilde{A}_{p}} \leq\|\varphi\|_{\left(\widetilde{A}_{p}\right)^{*}}\|f\|_{L^{p}}\|g\|_{L^{p^{\prime}}}
$$

for all $g \in \mathcal{S}\left(\mathbb{R}^{n}\right)$, it follows that $L_{f}^{(j)} \in L^{p^{\prime}}\left(\mathbb{R}^{n}\right)^{*}$ and its norm satisfies $\left\|L_{f}^{(j)}\right\|_{\left(L^{p^{\prime}}\right)^{*}} \leq\|\varphi\|_{\left(\widetilde{A}_{p}\right)^{*}}\|f\|_{L^{p}}$. Since $L^{p^{\prime}}\left(\mathbb{R}^{n}\right)^{*}=L^{p}\left(\mathbb{R}^{n}\right)$, we can find $h_{f}^{(j)} \in$ $L^{p}\left(\mathbb{R}^{n}\right)$ such that $\left\|h_{f}^{(j)}\right\|_{L^{p}}=\left\|L_{f}^{(j)}\right\|_{\left(L^{p^{\prime}}\right)^{*}}$ and

$$
L_{f}^{(j)}(g)=\int_{\mathbb{R}^{n}} h_{f}^{(j)}(x) g(x) d x \quad \text { for all } g \in \mathcal{S}\left(\mathbb{R}^{n}\right) .
$$

We define a linear operator $T_{j}$ from $\mathcal{S}\left(\mathbb{R}^{n}\right)$ to $L^{p}\left(\mathbb{R}^{n}\right)$ by $T_{j} f=\left(h_{f}^{(j)}\right)^{\vee}$. Then we have

$$
\left\|T_{j} f\right\|_{L^{p}}=\left\|\left(h_{f}^{(j)}\right)^{\vee}\right\|_{L^{p}}=\left\|L_{f}^{(j)}\right\|_{\left(L^{p^{\prime}}\right)^{*}} \leq\|\varphi\|_{\left(\widetilde{A}_{p}\right)^{*}}\|f\|_{L^{p}}
$$

for all $f \in \mathcal{S}\left(\mathbb{R}^{n}\right)$. That is, $T_{j}$ is bounded on $L^{p}\left(\mathbb{R}^{n}\right)$. Since $\tau_{x} f * g\left(2^{j} \cdot\right)=$ $f * \tau_{x} g\left(2^{j} \cdot\right)$, the equations

$$
\varphi\left(\tau_{x} f * g\left(2^{j} \cdot\right)\right)=L_{\tau_{x} f}^{(j)}(g)=\int_{\mathbb{R}^{n}} T_{j}\left[\tau_{x} f\right](y) g(-y) d y
$$

and

$$
\varphi\left(f * \tau_{x} g\left(2^{j} \cdot\right)\right)=L_{f}^{(j)}\left(\tau_{x} g\right)=\int_{\mathbb{R}^{n}} T_{j} f(y)\left[\tau_{x} g\right](-y) d y
$$

give $T_{j} \tau_{x}=\tau_{x} T_{j}$. Since $T_{j}$ is bounded on $L^{p}\left(\mathbb{R}^{n}\right)$ and commutes with translations, by [11, Chapter 1 , Theorem 3.16], we can find $m_{j} \in L^{\infty}\left(\mathbb{R}^{n}\right)$ such that $T_{m_{j}}=T_{j}$. We next show that $m_{j}=m_{0}\left(2^{j} \cdot\right)$ for all $j \in \mathbb{Z}$. Since $f * g\left(2^{j} \cdot\right)=\left[f_{2^{-j}} * g\left(2^{j} \cdot\right)\right]\left(2^{0} \cdot\right)$, the equations

$$
\varphi\left(f * g\left(2^{j} \cdot\right)\right)=L_{f}^{(j)}(g)=\int_{\mathbb{R}^{n}} T_{m_{j}} f(x) g(-x) d x
$$

and

$$
\begin{aligned}
& \varphi\left(\left[f_{2^{-j}} * g\left(2^{j} \cdot\right)\right]\left(2^{0} \cdot\right)\right)=L_{f_{2-j}}^{(0)}\left(g\left(2^{j} \cdot\right)\right) \\
& \quad=\int_{\mathbb{R}^{n}} T_{m_{0}} f_{2^{-j}}(x) g\left(-2^{j} x\right) d x=\int_{\mathbb{R}^{n}} T_{m_{0}\left(2^{j} \cdot\right)} f(x) g(-x) d x
\end{aligned}
$$

give $m_{j}=m_{0}\left(2^{j} \cdot\right)$. We write $m=m_{0}$. Then we have

$$
\varphi\left(f * g\left(2^{j} \cdot\right)\right)=\int_{\mathbb{R}^{n}} T_{m\left(2^{j} \cdot\right)} f(x) g(-x) d x=T_{m\left(2^{j} \cdot\right)} f * g(0)
$$

for all $f, g \in \mathcal{S}\left(\mathbb{R}^{n}\right)$ and $j \in \mathbb{Z}$. To show $m \in \max M_{p}\left(\mathbb{R}^{n}\right)$, we define a space $S$ by $S=\left\{\left\{g_{j}\right\}_{j \in \mathbb{Z}} \subset \mathcal{S}\left(\mathbb{R}^{n}\right):\left\{g_{j}\right\}_{j \in \mathbb{Z}}\right.$ is a finitely supported sequence $\}$. We note that, if $f \in \mathcal{S}\left(\mathbb{R}^{n}\right)$ and $\left\{g_{j}\right\}_{j \in \mathbb{Z}} \in S$, then $\sum_{j \in \mathbb{Z}} f * g_{j}\left(2^{j} \cdot\right) \in \widetilde{A}_{p}\left(\mathbb{R}^{n}\right)$ 
and $\left.\| \sum_{j \in \mathbb{Z}} f * g_{j}\left(2^{j} \cdot\right)\right)\left\|_{\widetilde{A}_{p}} \leq\right\| f\left\|_{L^{p}}\right\|\left\{g_{j}\right\}_{j} \|_{L^{p^{\prime}}\left(\mathbb{R}^{n}, \ell^{1}(\mathbb{Z})\right)}$. For $f \in \mathcal{S}\left(\mathbb{R}^{n}\right)$, we can define a linear functional $L_{f}$ on the dense subspace $S$ of $L^{p^{\prime}}\left(\mathbb{R}^{n}, \ell^{1}(\mathbb{Z})\right)$ by $L_{f}\left(\left\{g_{j}\right\}_{j}\right)=\varphi\left(\sum_{j \in \mathbb{Z}} f * g_{j}\left(2^{j} \cdot\right)\right)$ for $\left\{g_{j}\right\}_{j \in \mathbb{Z}} \in S$. From the boundedness of $\varphi$, it follows that

$$
\begin{aligned}
\left|L_{f}\left(\left\{g_{j}\right\}_{j}\right)\right| & \left.\leq\|\varphi\|_{\left(\widetilde{A}_{p}\right)^{*}} \| \sum_{j \in \mathbb{Z}} f * g_{j}\left(2^{j} \cdot\right)\right) \|_{\widetilde{A}_{p}} \\
& \leq\|\varphi\|_{\left(\widetilde{A}_{p}\right)^{*}}\|f\|_{L^{p}}\left\|\left\{g_{j}\right\}_{j}\right\|_{L^{p^{\prime}}\left(\mathbb{R}^{n}, \ell^{1}(\mathbb{Z})\right)}
\end{aligned}
$$

for all $\left\{g_{j}\right\}_{j \in \mathbb{Z}} \in S$, so that $L_{f} \in L^{p^{\prime}}\left(\mathbb{R}^{n}, \ell^{1}(\mathbb{Z})\right)^{*}$ and $\left\|L_{f}\right\|_{L^{p^{\prime}}\left(\mathbb{R}^{n}, \ell^{1}(\mathbb{Z})\right)^{*}} \leq$ $\|\varphi\|_{\left(\widetilde{A}_{p}\right)^{*}}\|f\|_{L^{p}}$. By the duality $L^{p^{\prime}}\left(\mathbb{R}^{n}, \ell^{1}(\mathbb{Z})\right)^{*}=L^{p}\left(\mathbb{R}^{n}, \ell^{\infty}(\mathbb{Z})\right)$, we can find $\left\{h_{j}\right\}_{j \in \mathbb{Z}} \in L^{p}\left(\mathbb{R}^{n}, \ell^{\infty}(\mathbb{Z})\right)$ such that $\left\|\left\{h_{j}\right\}_{j}\right\|_{L^{p}\left(\mathbb{R}^{n}, \ell^{\infty}(\mathbb{Z})\right)}=\left\|L_{f}\right\|_{L^{p^{\prime}}\left(\mathbb{R}^{n}, \ell^{1}(\mathbb{Z})\right)^{*}}$ and

$$
L_{f}\left(\left\{g_{j}\right\}_{j}\right)=\int_{\mathbb{R}^{n}} \sum_{j \in \mathbb{Z}} h_{j}(x) g_{j}(x) d x \quad \text { for all }\left\{g_{j}\right\}_{j} \in S .
$$

Now, (4) and

$$
\varphi\left(f * g\left(2^{j_{0}} \cdot\right)\right)=L_{f}\left(\left\{\delta_{j, j_{0}} g\right\}_{j}\right)=\int_{\mathbb{R}^{n}} h_{j_{0}}(x) g(x) d x
$$

give $T_{m\left(2^{\left.j_{0} .\right)}\right.} f=h_{j_{0}}^{\vee}$ for all $j_{0} \in \mathbb{Z}$, where $\delta_{j, j_{0}}=1$ if $j=j_{0}$ and $\delta_{j, j_{0}}=0$ if $j \neq j_{0}$. So, we get

$$
\begin{aligned}
& \left\|M_{m} f\right\|_{L^{p}}=\left\|\left\{T_{m\left(2^{j} .\right)} f\right\}_{j}\right\|_{L^{p}\left(\mathbb{R}^{n}, \ell^{\infty}(\mathbb{Z})\right)}=\left\|\left\{h_{j}^{\vee}\right\}_{j}\right\|_{L^{p}\left(\mathbb{R}^{n}, \ell^{\infty}(\mathbb{Z})\right)}
\end{aligned}
$$

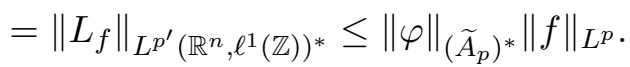

That is, $m \in \max M_{p}\left(\mathbb{R}^{n}\right)$. Finally, we prove $\varphi_{m}=\varphi$. Let $f \in \mathcal{S}\left(\mathbb{R}^{n}\right)$ and $\left\{g_{j}\right\}_{j} \subset \mathcal{S}\left(\mathbb{R}^{n}\right)$ satisfy $\|f\|_{L^{p}} \sum_{j \in \mathbb{Z}}\left\|g_{j}\right\|_{L^{p^{\prime}}}<\infty$. We note that $\sum_{j \in \mathbb{Z}} f *$ $g_{j}\left(2^{j} \cdot\right) \in \widetilde{A}_{p}\left(\mathbb{R}^{n}\right)$. Since $\sum_{|j| \leq N} f * g_{j}\left(2^{j} \cdot\right) \rightarrow \sum_{j \in \mathbb{Z}} f * g_{j}\left(2^{j} \cdot\right)$ in $\widetilde{A}_{p}\left(\mathbb{R}^{n}\right)$ as $N \rightarrow \infty$, using the continuity and linearity of $\varphi$ and (4), we have

$$
\begin{aligned}
\varphi\left(\sum_{j \in \mathbb{Z}} f * g_{j}\left(2^{j} \cdot\right)\right) & =\lim _{N \rightarrow \infty} \sum_{|j| \leq N} \varphi\left(f * g_{j}\left(2^{j} \cdot\right)\right) \\
= & \lim _{N \rightarrow \infty} \sum_{|j| \leq N} T_{m\left(2^{j} \cdot\right)} f * g_{j}(0)=\sum_{j \in \mathbb{Z}} T_{m\left(2^{j} \cdot\right)} f * g_{j}(0) .
\end{aligned}
$$

Let $f=\sum_{i \in \mathbb{N}} \sum_{j \in \mathbb{Z}} f * g_{j}\left(2^{j} \cdot\right) \in \widetilde{A}_{p}\left(\mathbb{R}^{n}\right)$, where $\left\{f_{i}\right\},\left\{g_{i, j}\right\}_{i, j} \subset \mathcal{S}\left(\mathbb{R}^{n}\right)$ satisfy $\sum_{i \in \mathbb{N}} \sum_{j \in \mathbb{Z}}\left\|f_{i}\right\|_{L^{p}}\left\|g_{i, j}\right\|_{L^{p^{\prime}}}<\infty$. Since

$$
\sum_{i \in \mathbb{N}}\left\|f_{i}\right\|_{L^{p}}\left\|\left\{g_{i, j}\right\}_{j}\right\|_{L^{p^{\prime}}\left(\mathbb{R}^{n}, \ell^{1}(\mathbb{Z})\right)} \leq \sum_{i \in \mathbb{N}} \sum_{j \in \mathbb{Z}}\left\|f_{i}\right\|_{L^{p}}\left\|g_{i, j}\right\|_{L^{p^{\prime}}}<\infty,
$$

we see that $\sum_{i \leq N} \sum_{j \in \mathbb{Z}} f * g_{j}\left(2^{j}.\right) \rightarrow f$ in $\widetilde{A}_{p}\left(\mathbb{R}^{n}\right)$ as $N \rightarrow \infty$. Hence, from 
the continuity and linearity of $\varphi$ and (5), we get

$$
\begin{aligned}
\varphi(f) & =\lim _{N \rightarrow \infty} \sum_{i \leq N} \varphi\left(\sum_{j \in \mathbb{Z}} f_{i} * g_{i, j}\left(2^{j} \cdot\right)\right) \\
& =\lim _{N \rightarrow \infty} \sum_{i \leq N} \sum_{j \in \mathbb{Z}} T_{m\left(2^{j} .\right)} f_{i} * g_{i, j}(0)=\varphi_{m}(f) .
\end{aligned}
$$

The proof is complete.

Acknowledgments. The author gratefully acknowledges helpful discussions with Professor Eiichi Nakai and Professor Kôzô Yabuta.

\section{References}

[1] N. Asmar, E. Berkson and T. A. Gillespie, Summability methods for transferring Fourier multipliers and transference of maximal inequalities, in: Analysis and Partial Differential Equations: A Collection of Papers Dedicated to Mischa Cotlar, ed. by C. Sadosky, Lecture Notes in Pure and Appl. Math. 122, Dekker, New York, 1990, $1-34$.

[2] E. Berkson, M. Paluszyński and G. Weiss, Wavelet decompositions of Fourier multipliers, Proc. Amer. Math. Soc. 125 (1997), 2395-2399.

[3] M. Christ, L. Grafakos, P. Honzík and A. Seeger, Maximal functions associated with Fourier multipliers of Mikhlin-Hörmander type, Math. Z. 249 (2005), 223-240.

[4] H. Dappa and W. Trebels, On maximal functions generated by Fourier multipliers, Ark. Mat. 23 (1985), 241-259.

[5] J. Duoandikoetxea, Fourier Analysis, Grad. Stud. Math. 29, Amer. Math. Soc., Providence, RI, 2001.

[6] A. Figà-Talamanca, Translation invariant operators in $L^{p}$, Duke Math. J. 32 (1965), 495-501.

[7] A. Figà-Talamanca and G. I. Gaudry, Multipliers of $L^{p}$ which vanish at infinity, J. Funct. Anal. 7 (1971), 475-486.

[8] M. Jodeit, Restrictions and extensions of Fourier multipliers, Studia Math. 34 (1970), 215-226.

[9] C. Kenig and P. Tomas, Maximal operators defined by Fourier multipliers, ibid. 68 (1980), 79-83.

[10] R. Larsen, An Introduction to the Theory of Multipliers, Springer, Berlin, 1971.

[11] E. M. Stein and G. Weiss, Introduction to Fourier Analysis on Euclidean Spaces, Princeton Univ. Press, Princeton, 1971.

[12] H. Triebel, Theory of Function Spaces, Birkhäuser, Basel, 1983.

Department of Mathematics

Graduate School of Science

Osaka University

Toyonaka, Osaka 560-0043, Japan

E-mail: tomita@gaia.math.wani.osaka-u.ac.jp 